\title{
Key Business Competencies for New Aerospace Engineers
}

\author{
Clair J. Nixon \\ Associate Dean \\ Mays Business School \\ Texas A\&M University
}

\section{Introduction}

Curricula in most engineering schools fail to provide adequate training and development of future engineers in regards to basic business principles. The newly minted engineers generally have excellent technical skills, but lack an understanding of the key business principles that drive the aerospace industry. After nearly 200 interviews of aerospace engineers in the workforce, four key business competencies emerged as the most important skills for new engineer hires. These four competencies are as follows:

- Strong communications skills

- Enhanced financial acumen

- Better understanding of the customer

- Comprehension of the life cycle of a product

There were clearly other business competencies that would be desirable for new engineers. However, many of these business competencies would likely be used later in an engineer's career. For the purposes of this presentation, I will focus on the above four competencies.

There has been considerable discussion in the literature relative to alternative instructional methods for enhancing engineering education. In some cases, the course development and delivery has been developed with consideration of ABET criteria with special focus on requirement $3(\mathrm{~g})$ [1]. Other approaches to engineering education have included interdisciplinary course development [2]. The use of capstone courses has likewise become a popular medium for integrating business principles into the engineering curriculum [3]. What is missing, however, is a systematic approach to covering the basics of business education for aerospace engineering students.

\section{Process}

As a part of the Boeing Corporation Welliver Faculty Fellowship Program (Welliver), faculty members are encouraged to submit proposals for an eight-week summer program. The proposals are generally technical in nature with the faculty member desiring to learn more about specific components or systems within the Boeing organization. Historically, most of the participants in the program have been faculty associated with engineering or computer science departments. There have been, however, 
several business school faculty members that have been successful in having their proposals accepted and thereby allowed participation in the program. As such, the proposal from the author focused on examining what the key business competencies should be for recently minted engineering school graduates entering the aerospace workforce.

Prior to accepting the invitation to participate in the Welliver Program, the author co-taught a capstone aerospace design course in which business principles and applications were stressed. Several lectures and exercises utilized financial information of the aerospace industry and included analysis of specific aerospace projects. It was assumed that the students had little to no prior exposure to basic business principles. Class exercises were used to determine the level of competency in applying these business principles to engineering problems. The design of the interview instrument used in the Welliver Program was determined after the completion of this course and was based in part on the perceptions of the value of the information for these university students. Further, multiple discussions were held with engineering and business faculty as well as Boeing personnel prior to the final development of the interview instrument and the start of the interview period.

Over a seven-week interview period, nearly two hundred engineers, accountants, finance personnel, human resource and management associates and others within Boeing were interviewed. The questions to be answered by the interviewees were the same as designed by the author prior to the initiation of the plant visits. The interviewees ranged in experience from interns to 35 -year veterans with the firm. Further, management experience ranged from the new interns to executive vice presidents. Although most of the interviews occurred on a one-on-one basis, there were several instances in which a group of eight to ten individuals participated in the same interview session. The author was charged with taking copious notes of the discussions. Literally hundreds of pages of notes were collected from the interviews over the course of the seven weeks.

The locations of the interviews varied both geographically and by product line. Interviews were held in Anaheim, CA, Canoga Park, CA, Long Beach, CA, Seal Beach, CA, St. Louis, Missouri, Clear Lake, Texas, Everett, WA, and Seattle, WA. The scope of the business activities represented by the interviewees included: future combat systems, space shuttle main engine, commercial aircraft, C-17 transport, fighter aircraft, missiles and other NASA projects. In addition to the Boeing employees, faculty from several universities as well as practicing engineers from a number of different firms including Bechtel, Parsons and Rockwell were also interviewed.

After completion of seven weeks of interviews, a presentation of the results was made to Boeing representatives in St. Louis. The focus of the presentation was two-part, namely, identification of key business competencies for the new engineers and a model for a new employee orientation program. Both Boeing representatives and the other Boeing Welliver Fellows provided a critique of the presentation. The results below include their comments and reflections. 


\section{Results}

As expected, there was a diversity of opinion concerning the most valuable business competencies to enhance the potential for success among new engineers. In fact, there were fifteen competencies identified by the interviewees. These competencies included:

- Communication skills

- Critical thinking

- Earned value concepts

- Scheduling of projects

- Cost of capital

- Capital investment analysis

- Profit and loss statements

- Project budgeting

- Marketing

- Basic management skills

- Project negotiations

- Team work and working in teams

- Business ethics

- Maximizing shareholder value

- Product life cycle

It was clear that some of the above skills could be grouped together. However, four key competencies consistently emerged from the interviews. These key competencies, identified above, were also ranked ordered by the interviewees. The following elaboration of these key competencies is in the order of their perceived importance by the majority of the interviewees. In all cases, good communications skills were included as an important competency for advancement within the business. In fact, some of the respondents indicated that if new hires had only good communication skills, combined with strong technical engineering skills, they would be successful in the business.

All interviewees identified the lack both written and verbal communication skills as of greatest concern among new engineers. Although there was recognition that the communication skills had improved in recent years, there was a consistent perception that new engineers lacked presentation skills, especially in small group settings. There was no question that the new engineers could maneuver around a PowerPoint presentation. The problem is that the content was lacking. There was also a concern over focus in how the presentations were packaged. There was overwhelming encouragement for enhanced requirements in the college classroom for more oral presentations critiqued by industry personnel. There was also the recognition that current college students are immersed, to a much great extent than in the past, with team exercises and presentations. Nonetheless, there was a greater concern about strategic thinking in the presentations than the actual presentations themselves.

It was deemed highly valuable for upward mobility in the aerospace industry that young engineers be able to provide a briefing. They should be able to clearly and 
concisely present a message. They also need to know when to say they do not know the answer. A common failing among new engineers was overstepping personal knowledge. In an attempt to not appear unprepared, new engineers often extrapolated beyond the data.

Further, as a part of communication skills, it was also deemed valuable to learn negotiation skills. Negotiation skills were considered important in both internal and external discussions. New engineers would not likely get involved in too many external financial negotiations, but there are a number of opportunities for negotiating work requirements for specific projects. In addition, being able to work in a team environment requires the ability to come to consensus on both job responsibility and accountability. Good negotiation skills enable the associate to better understand both sides of the problem and seek for resolution in an expeditious manner. The interviewees indicated that they did not receive any training in negotiation skills unless they had pursued a graduate degree in business. Especially invaluable would have conflict resolution skills in a team environment. It was generally agreed among the interviewees that a considerable amount of time and resources could be saved through implementing good conflict resolution practices. For many of these interviewees, understanding how to negotiate would have been an invaluable asset during their tenure in the industry.

Closely aligned with good negotiation skills was gaining a greater appreciation of different cultures. In a global business environment, especially exhibited in the aerospace industry, understanding how businesses operate in different countries and cultures potentially forestalls missteps in business transactions. There was definitely an encouragement for new engineers to have some level of cultural awareness and how business operates in different cultural environments.

Written communication was also deemed a critical component of the business skills of new engineers. Being able to discuss highly technical issues in a clear and concise manner was perceived as generally lacking among new engineers. From a business perspective, the focus was on writing short memorandums. With the proliferation of electronic mail, the interviewees suggested that little thought is often employed in the development of an email. The result is often confusion because the sender did not fully develop the thoughts of the message.

It was suggested by most of the interviewees that a greater emphasis on written and oral communication would provide new engineers with enhanced management opportunities within the aerospace industry. Those individuals who have tended to rise to levels of significant management have possessed both written and oral communication skills. In fact, the lack of such skills was perceived a clear inhibitor to advancement within the industry.

The final issue related to communications skills was that all engineers, irrespective of their position in the firm, needed to demonstrate good written and oral communication. Several interviewees commented that they had no interest in firm management, but still recognized that good communication skills were critical to success 
at all levels of responsibility. It was clear that the ability to communication effectively cut across all positions within Boeing.

The second important skill as identified by 85 percent of the respondents was determined to be increased financial acumen. Financial acumen is being able to both understand and solve business problems. The key financial skills identified by the interviewees fell into three main categories. First, there needs to be a good understanding of the basic relationship between income statement and balance sheet items. Better comprehension of these accounting principles will enable the engineer to assess the financial returns to the firm through good project management. Although the new engineer will not likely have profit and loss responsibility early in a career, an understanding of the principles was deemed important for continued success of business opportunities. It was considered important to have a basic understanding of what is included in the these financial statements and how performance in the individual businesses impacts on the firms' ability to succeed. It was not deemed important for the engineers to have an in-depth knowledge of the accounting principles until they became project managers or at similar levels within a large firm. On the other hand, for those engineers that would be entering the workforce at a small engineering firm, an understanding of the basic accounting principles was considered of greater importance. A number of the interviewees in the sample had joined Boeing after anywhere from one to ten years of experience in small, medium and large firms. For those who had previously been employed in a small firm setting, there was the recognition that basic financial acumen was more important early in the engineer's career than those employed by large firms. In the small firms, the new engineer often was required to complete multiple tasks within projects, including budgeting and other financial reporting requirements. As a result, such individuals would likely be required to utilize their accounting knowledge much sooner than those employed by large firms.

Another important financial skill for new engineers is understanding the basics of capital investment analysis. The principle of time value of money is not a focus during the technical engineering education. However, in practice, understanding that money leaving the firm today is more valuable than the same amount of money flowing back in future periods. In the aerospace industry most of the capital projects are for a multi-year period. If the potential returns are too far in the future, the value of those in relation to the money being spent today may render the project inviolate. New engineers should be able to apply the capital investment principles no matter the size of project.

The third financial principle identified by the interviewees was critical is budgeting. Even on the most basic projects, the engineer needs to understand how the budget was put together and what is available for the particular project. There was clear agreement that engineers do not concern themselves, as they should, with costs. There is a general lack of accountability in the profession regarding project cost. The engineering mentality, according to the interviewees, is that whatever it takes to get it right is what is important. Often, however, the engineers have a difficult time staying within the budget because they don't follow the requirements of the project and often over engineer the item. Being able to understand basic budgeting principles would enable the new engineer 
to focus on completing projects within the cost constraints imposed. It was often expressed by the interviewees that many engineers believe it is the accountants' responsibility to watch the costs. In order for engineers to be successful in the engineering business, they must be accountable for costs as well. These principles need to be more clearly presented to new engineers.

The third category of developing a better understanding of the customer was determined to be important by over 75 percent of the respondents. It is important that the engineer understand that there are generally multiple customers in most businesses. These include the purchaser of the product or service (i.e., the airlines or government), the ultimate benefactor of the product or service (passenger or taxpayer), the associates within the company, the shareholder, and often the supplier. Each of these "customers" has a different set of goal and requirements for measuring success. It was determined that new engineers would be greatly benefited by having exposure to the different customers that they will likely deal with. In many cases, the interviewees suggested that engineers do not realize that they must satisfy customers on several different levels. It is not just completing a single project that is important. Rather, it is all about building long-term relationships so that opportunities for future projects will be realized.

There is often the misconception that the customers include only those to whom we sell our product or service. On the contrary, a customer is any entity with whom we have a business relationship. For example, the failure to treat the supplier as a "customer" may lead to less than optimal business results. Such a situation would occur if the entity needs a special consideration on the shipment of a critical component to business operation. Treating the supplier in similar manner to other customers will often mitigate potential supply problems Further, stakeholders or shareholders are also customers because they are the ultimate owners of the company. Failure to recognize their critical role in the business may undermine the ability of management to effectively move the company forward.

The fourth category, which is a comprehension of the life cycle of a product, was recognized by over half of the respondents as one of the top four business competencies. This cycle includes the following phases: market analysis, design phase, building the initial product, production phases and support services over the life of the product.

The first phase of the life cycle of the product, the strategy, is divided into several components. The new engineer should have a good understanding of how the strategy is determined. For example, the strategy will generally include the business objectives, opportunities available, state of current products and competition. Each of these factors is important in developing a strategy around a potential product.

The second phase in the life cycle is preliminary design. Key business principles are linked to good engineering practices to develop a good design. The preliminary design requires that the engineers talk to customers, benchmark competition, assist the financing personnel in understanding financial requirements, look to the scheduling of the project and be able to ascertain the various types of risks associated with the project. the 
engineer applies technical engineering knowledge with good business principles. There is the detail design, budgets to follow, cash flow implications and other issues.

The third phase is to build and deliver the product. Key business principles in this phase include managing the production process for costs and returns, reacting to sales and customer needs and identifying opportunities and problems.

Finally, the engineer has the responsibility to maintain the product that has been developed. In many cases, the maintenance of the product will require up to 50 years of service. There are also requirements for other support and upgrades of the product.

At each step in the life cycle the engineer must be able to review and assess the financial and other business implications of the project. The interviewees were adamant that there is not enough long-term business understanding on the part of new engineers. There is too much short-term focus from a financial perspective. With a thorough comprehension of the linkage between the business and the engineering sides of the operation, the new engineer can be a more valuable employee to the firm.

\section{Summary}

It is not expected that new engineers will have in-depth knowledge of all of the above business principles. Rather, the basic business principles should be couched in relevant case studies so that the engineers can relate to them as they move through their engineering careers. It is incumbent upon both the academic community and the employers that there be a clarion call for better business acumen for the new engineer. The panacea is often the MBA. However, the opportunity to more significantly contribute to the well-being of the business as a new engineer is an important goal especially for the highly motivated.

What has been outlined above may serve as a model for both firms and academia. Although there are a number of places in academia where some of these business principles are being addressed, there is still an inadequate response to the need. There are several institutions that now provide capstone courses with a focus on the application of business principles to an engineering problem. This is especially true in some of the larger aerospace departments with linkages to their business schools. Unfortunately, the academics too often contend that the curriculum is already too congested with technical courses. It is suggested that the university community examine their position and evaluate the trade-off between additional technical courses and a better understanding of business applications. The author believes that the academic community has a responsibility to better equip its newly minted engineers with the "basics of business."

In the circumstances where new employees do not have the financial skills addressed above, firms are encouraged to provide some level of supplemental training. It is suggested that the new employee orientation is a critical opportunity to emphasize these business principles. Most of the business principles could be developed around a business case for the specific products being produced. A two-day business/engineering 
orientation case, built around the product life cycle concept, would go a long way toward helping the new engineer understand and the importance of basic business principles. 


\section{References}

[1] R. Felder and R. Brent. (2003). Designing and Teaching Courses to Satisfy the ABET Engineering Criteria. The Journal of Engineering Education. 92(1):725.

[2] O’Connor, T, H. Sibray, and K. Forinash. (2001). Interdisciplinary Research Project Involving Physics and Electrical Engineering Students. The Journal of Engineering Education. 90(3):423-428.

[3] Wang, E. and J. Kleppe. (2001). Capstone Course Includes MBA Students New Product Development, Sales and Marketing. The Journal of Engineering Education. 90(4):565-569. 